\title{
Comparative Study of Antitumor Activity between Lipophilic Bismuth Nanoparticles (BisBAL NPs) and Chlorhexidine on Human Squamous Cell Carcinoma
}

\author{
Fernando Martínez-Pérez, ${ }^{1}$ Claudia María García-Cuellar, ${ }^{2}$ Rene Hernandez-Delgadillo (D), \\ Valentin Zaragoza-Magaña, ${ }^{1}$ Yesennia Sánchez-Pérez, ${ }^{2}$ Irene Meester $\mathbb{D}^{\mathbb{D}},{ }^{3}$ \\ Sergio Eduardo Nakagoshi-Cepeda, ${ }^{1}$ Juan Manuel Solís-Soto ${ }^{1 D}{ }^{1}$ \\ María Argelia Akemi Nakagoshi-Cepeda, ${ }^{1}$ Shankararaman Chellam, ${ }^{4}$ \\ and Claudio Cabral-Romero ${ }^{1}{ }^{1}$ \\ ${ }^{1}$ Universidad Autónoma de Nuevo León, UANL, Facultad de Odontología, Laboratorio de Biología Molecular, Monterrey, \\ Nuevo León, Mexico \\ ${ }^{2}$ Subdirección de Investigación Básica, Instituto Nacional de Cancerología, Ciudad de México, Mexico \\ ${ }^{3}$ Universidad de Monterrey, Departamento de Ciencias Básicas, San Pedo Garza García, Mexico \\ ${ }^{4}$ Texas A\&M University, TX, USA
}

Correspondence should be addressed to Claudio Cabral-Romero; claudio.cabralrm@uanl.edu.mx

Received 2 August 2019; Revised 10 October 2019; Accepted 26 October 2019; Published 5 December 2019

Academic Editor: Ruibing Wang

Copyright (c) 2019 Fernando Martínez-Pérez et al. This is an open access article distributed under the Creative Commons Attribution License, which permits unrestricted use, distribution, and reproduction in any medium, provided the original work is properly cited.

\begin{abstract}
The objective of this study was to compare the antitumor activity of lipophilic bismuth nanoparticles (BisBAL NPs) and chlorhexidine (CHX) on human squamous cell carcinoma. BisBAL NPs were synthesized by colloidal method and characterized by energy dispersive X-ray spectroscopy in conjunction with scanning electron microscopy (EDS-SEM). The effect of BisBAL NPs and CHX on oral cancer cell line (CAL-27) and nontumor control cell human gingival fibroblasts (HGFs) was determined by MTT cell viability assay. The obtained results showed selective inhibition of CAL-27 cell growth by BisBAL nanoclusters. A $24 \mathrm{~h}$ exposition to $25 \mu \mathrm{M}$ BisBAL NP decreased 91\% of CAL-27 cell growth, while nontumor HGFs cells were unaffected by BisBAL NPs showing $90 \%$ of cell viability. In contrast, CHX kills both CAL-27 and HGFs with the same efficacy. $25 \mu \mathrm{M}$ of CHX decreased $97 \%$ and $80 \%$ of tumor and nontumoral cell growth. BisBAL NP and CHX alter cell permeability suggesting that action mechanism may include loss of cell membrane integrity. Also, CHX and not BisBAL NP presented genotoxicity on genomic DNA of tumor cells. As conclusion, BisBAL NPs have a selective antitumor activity on human squamous cell carcinoma, unlike CHX which was cytotoxic for both tumoral and nontumoral control cells.
\end{abstract}

\section{Introduction}

Head and neck squamous cell carcinomas (HNSCC) are important public health challenges worldwide, being the $11^{\text {th }}$ most common cancer detected with approximately 50-300, 000 new cases each year $[1,2]$. It is expected that 10,030 people die in 2018 due to these cancers only in the USA, but the number could be higher in less developed regions [2]. Different risk factors have been described like alcohol, smoking, human papillomavirus (HPV), dietary deficiencies, and fungal infections [3]. HNSCC are progressive chronic diseases in which healthy epithelium is modified into a carcinogenic tissue through one or more risk factors in each specific individual. HNSCC are more frequent in men than in women and their prognosis is poor, especially in late diagnosis [4].

Although chemotherapy is an important treatment option for most cancers after surgery, it has several limitations, amongst which are a lack of target specificity, the development 
of resistance, and severe side effects [5]. For example, the side effects of the commonly used anticancer agent cisplatin (CIS) include nephrotoxicity, neurotoxicity, bone marrow suppression, and vomiting $[6,7]$. The use of the anticancer drug, docetaxel (DCT), is limited due to liver injury $[8,9]$, while doxorubicin (DOX) induces cardiotoxicity and resistance [10]. Unfortunately, these kinds of drugs encourage patients to leave chemotherapy due to their nondesired secondary effects. It is urgent to develop selective drugs for cancer treatment.

CHX is a broad-spectrum antimicrobial agent that is found as an active ingredient in mouthwash and is frequently used in dentistry to reduce bacterial load. Previous reports have described the in vitro antitumoral effect of CHX on breast and oral cancer cell lines [11, 12]. CHX competes effectively with positive drugs like Adriamycin; however, it is cytotoxic for nontumoral cells [13]. Earlier, it was described that CHX inhibits protein-protein interactions mediated by the antiapoptotic protein Bcl-xL at physiological concentrations inducing apoptosis in several tumor cell lines derived from the tongue and pharynx [14].

Nanomedicine is a recent discipline that uses nanotechnology to develop "smarter drugs" [15] that promise to be more efficient than traditional anticancer drugs [16]. Nanomedicine constitutes an interesting field to develop new antitumor drugs. In the last years, several reports have described the employment of different kinds of nanoparticles to treat cancer, underlining selenium, silver, and zinc nanoparticles and nanoformulations for drug delivery [17-20]. Earlier, our group described the selective antitumor activity of lipophilic bismuth nanoparticles (BisBAL NPs) on breast human cancer cells showing a specific and dose-dependent phenomenon [21]. However, it is unknown if BisBAL NPs are effective against oral cancer cells.

The aim of this study is to compare the antitumor effect of BisBAL NPs and CHX on human squamous cell carcinoma. Our hypothesis is that BisBAL NPs will be better as an antitumor agent than CHX based on their lipophilic and cationic properties.

\section{Material and Methods}

2.1. Synthesis and Characterization of BisBAL NPs. The BisBAL NPs were prepared by colloidal method like previously described [22]. Briefly, $0.485 \mathrm{~g}$ of $\mathrm{Bi}\left(\mathrm{NO}_{3}\right)_{3} \cdot 5 \mathrm{H}_{2} \mathrm{O}$ was dissolved in $20 \mathrm{~mL}$ propylene glycol, heated to $80^{\circ} \mathrm{C}$, and agitated for $2 \mathrm{~h}$ to obtain a $50 \mathrm{mM} \mathrm{Bi}^{3+}$ solution. A $2: 1$ molar ratio of $\mathrm{Bi}^{3+}$ (Bis) to 2,3-dimercapto-1-propanol (BAL) was prepared by adding $25 \mu \mathrm{L} 10 \mathrm{M}$ BAL to $10 \mathrm{~mL} 50 \mathrm{mM} \mathrm{Bi}^{3+}$ solution. The distribution of BisBAL NP's size and shape were analyzed with scanning electron microscopy (SEM; FEI Tecnai G2 Twin, Hillsboro, OR, USA; $160 \mathrm{kV}$ accelerating voltage). The specific presence of bismuth was corroborated by energy-dispersive X-ray spectroscopy (EDS) SEM (Oxford INCA X-Sight, Tubney Woods, UK).

2.2. Cell Culture. The human squamous cell carcinoma (CAL-27) was obtained from the American Type Culture Collection (ATCC CRL-2095; Rockville, MD, USA). The pri- mary culture of human gingival fibroblasts (HGFs) was used as a nontumor control cells. The cell were cultivated in Dulbecco's modified Eagle's Medium/Ham's F12 (DMEM/F12) supplemented with $10 \%$ fetal bovine serum (FBS; Gibco Invitrogen, Carlsbad, CA, USA), $100 \mathrm{U} / \mathrm{mL}$ penicillin, $100 \mu \mathrm{g} / \mathrm{mL}$ streptomycin, and $0.25 \mu \mathrm{g} / \mathrm{mL}$ amphotericin B (Sigma-Aldrich Corporation, St. Louis, MO, USA) in cell culture flasks (Corning Inc., Corning, NY, USA) at $37^{\circ} \mathrm{C}$ in a humidified atmosphere with $5 \% \mathrm{CO}_{2}$. Cells of a confluent monolayer were harvested by scraping, washed three times with $10 \mathrm{mM}$ phosphate-buffered saline, $\mathrm{pH} 7.4$ (PBS), and counted with a hemocytometer.

2.3. MTT Cell Viability Assay. The effect of BisBAL NPs on the viability of human squamous cell carcinoma (CAL-27) and nontumor control cells (HGFs) was evaluated using the 3-(4,5-dimethylthiazol-2-yl)-2,5-diphenyltetrazolium bromide (MTT) cell viability assay (Biotium, Hayward, CA, USA) $[21,23]$. Briefly, $1 \times 10^{5}$ cells were incubated $\left(24 \mathrm{~h}, 37^{\circ} \mathrm{C}, 5 \%\right.$ $\mathrm{CO}_{2}$ ) with $0,1,10,25,50$, and $100 \mu \mathrm{M}$ of BisBAL NP or CHX (Ultradent Products, South Jordan, UT) in solution and $10 \mu \mathrm{M}$ docetaxel (Zurich Pharma, Mexico city, Mexico) as a positive control. The treatment was terminated by washing the cells with PBS. Next, cells were incubated with MTT $\left(10 \mu \mathrm{L} /\right.$ well, $\left.2 \mathrm{~h}, 37^{\circ} \mathrm{C}, 5 \% \mathrm{CO}_{2}\right)$ in the dark according to the provider's instructions. Next, the medium was removed and $100 \mu \mathrm{L}$ dimethylsulfoxide (DMSO) was added to dissolve the reduced MTT formazan product. To quantify the reduced MTT, the absorbance at $570 \mathrm{~nm}\left(\mathrm{~A}_{570}\right)$ was measured with a microplate absorbance reader (BioTek, Winooski, VT); DMSO served as a blank. The assay was performed in triplicate to assess the veracity of results.

2.4. Cell Membrane Permeability by Fluorescence Microscopy. To explore the effect of BisBAL NPs and CHX on the cell membrane of tumor cells, intracellular calcein-AM assay [24] and fluorescence microscopy were employed. This assay was used to analyze the cell membrane permeability of CAL27 cells after a $24 \mathrm{~h}$ exposure to 0,10 , and $50 \mu \mathrm{M}$ of BisBAL $\mathrm{NP}$ and $50 \mu \mathrm{M} \mathrm{CHX}$; cells exposed to the pure culture medium, served as growth control. After incubation, cells were washed three times with PBS and stained with $2 \mu \mathrm{M}$ calcein-AM (Biotium, Hayward, CA, USA) and 4',6-diamidino-2-phenylindole (DAPI) (Abcam Inc., Cambridge, UK) for $30 \mathrm{~min}$. at $37^{\circ} \mathrm{C}$. Next, cells were washed again with PBS and air-dried in the dark. Cell morphology was observed with FITC and DAPI filters at $485 \mathrm{~nm}$ and $358 \mathrm{~nm}$, respectively (Thornwood, NY).

2.5. DNA Ladder Assays. To evaluate the possible genotoxic effect of BisBAL NP on tumor cells, DNA ladder assays were carried out following the protocol previously described [25]. CAL-27 cells were exposed for $24 \mathrm{~h}$ to $50 \mu \mathrm{M}$ BisBAL NPs and $50 \mu \mathrm{M}$ of $\mathrm{CHX}$, and as negative control, growing cells with only culture media were used. After treatment, tumor cells were collected and genomic DNA was extracted using Isolate II Genomic DNA Kit (Bioline, London, UK) following manufacturer's instructions. The obtained DNA samples were analyzed by electrophoresis in $1 \%$ agarose gel 


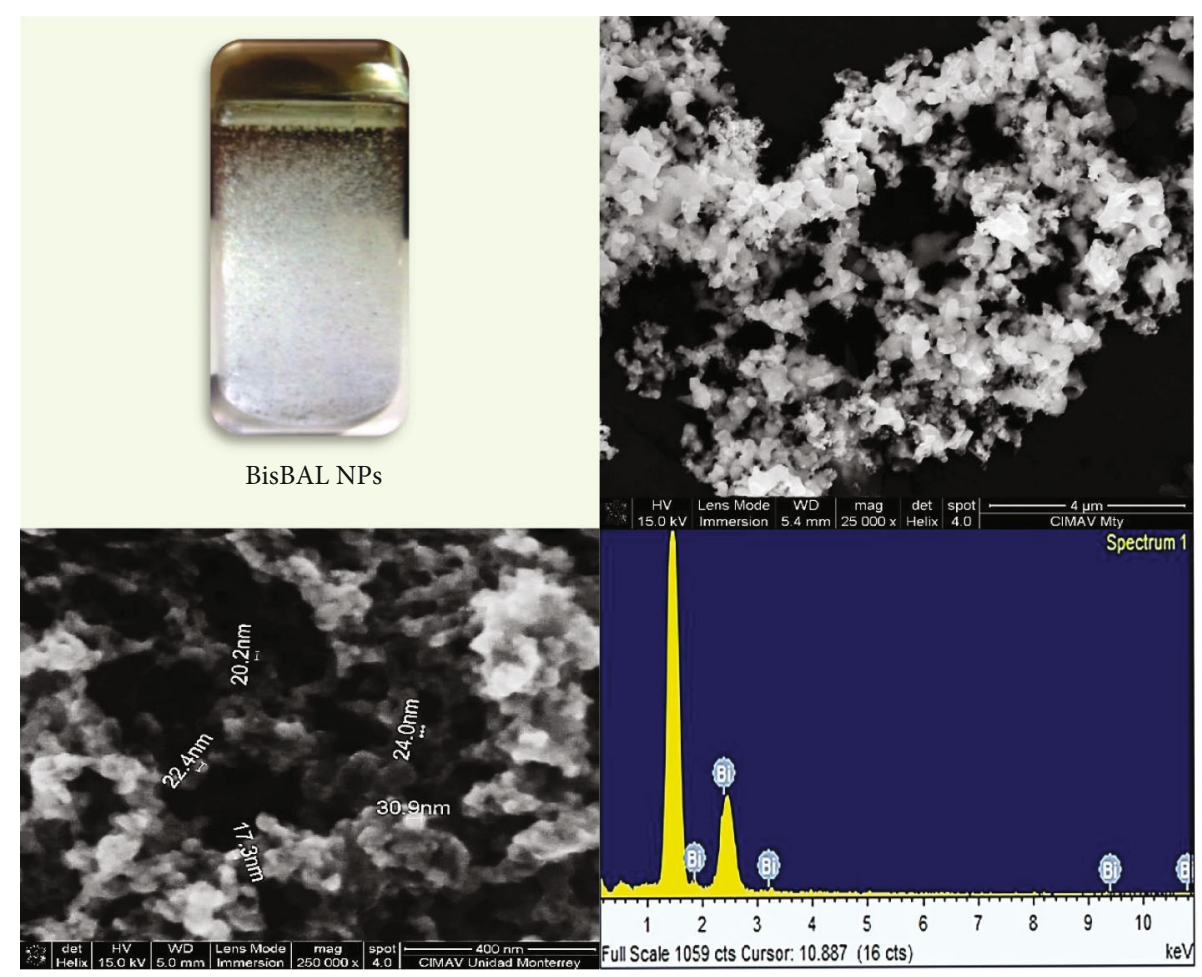

FIgURE 1: Characterization of BisBAL NPs. Shape, size, and distribution of BisBAL NPs were obtained by scanning electron microscopy (SEM). The specific presence of bismuth was confirmed by energy-dispersive X-ray spectroscopy (SEM-EDS).

and ethidium bromide staining. The presence of several DNA fragments (ladder shape) or a tail will indicate a damaged DNA.

2.6. Statistical Analysis. A multiple comparison 2-way ANOVA with Tukey's correction was used to compare among groups. For all statistical tests, a significance level of $\alpha=0.05$ was considered.

\section{Results}

3.1. Characterization of BisBAL NPs. The obtained BisBAL NPs were round in shape with an average diameter of $24 \mathrm{~nm}$ (Figure 1). Bismuth presence was specifically confirmed by EDS-SEM (Figure 1). The BisBAL NPs formed electrodense clusters, a typical characteristic of this kind of nanostructures (Figure 1). A fresh batch of BisBAL NPs was used to study the antitumor activity on human squamous cell carcinoma.

3.2. Antitumor Activity. BisBAL NPs inhibited $86 \%$ of the growth of the CAL-27 cell line since $10 \mu \mathrm{M}$ BisBAL NP after $24 \mathrm{~h}$ exposition (Figure 2). The highest reduction of tumor cell growth was obtained at $50-100 \mu \mathrm{M}$ BisBAL NP, with $90 \%$ of inhibition in comparison with growth control (Figure 2). On the other hand, control nontumor cell HGFs showed $84 \%$ of cell viability when they were exposed to $50 \mu \mathrm{M}$ BisBAL NP (Figure 2). The positive control docetaxel (a commercial antitumor drug) at a final concentration of $10 \mu \mathrm{M}$ inhibits $98 \%$ of tumor cell growth, but at the same time reduces $50 \%$ of nontumor cell HGFs growth. In the case

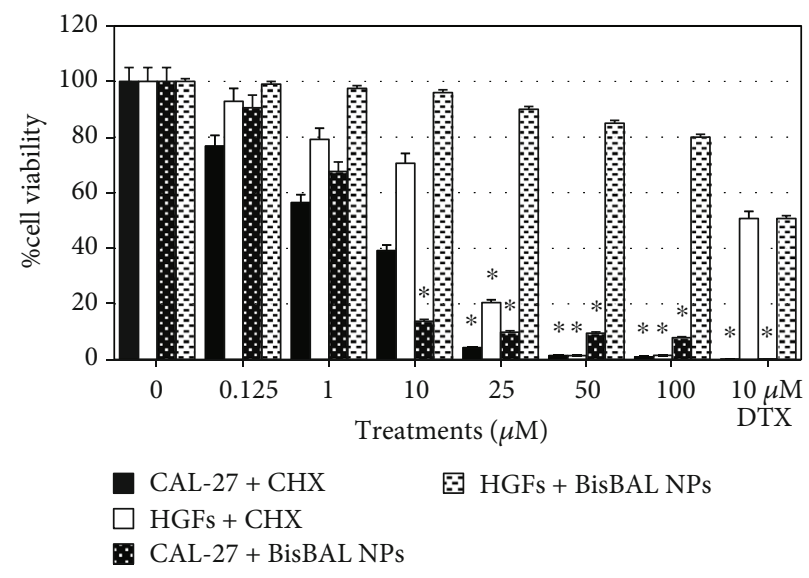

Figure 2: Antitumor effect of BisBAL NPs and CHX on CAL-27 and HGF cell lines. Cell viability was evaluated with the MTT cell viability assay after a $24 \mathrm{~h}$ exposure to $0,0.125,1,10,25,50$, and $100 \mu \mathrm{M}$ BisBAL NP or CHX and $10 \mu \mathrm{M}$ DTX (positive control of cytotoxicity). Readings were performed in triplicate. Results are representative of three independent experiments. After a multiple comparison 2-way ANOVA with Tukey's correction, asterisk indicates statistical differences $(p<0.0001)(\alpha=0.05)$. Error bars indicate mean $\pm \mathrm{SD}(n=7)$.

of $\mathrm{CHX}$, the highest reduction of CAL-27 cell growth was achieved at $25 \mu \mathrm{M}$ with a $97 \%$ decrease, but also inhibited $80 \%$ that of nontumor cells (Figure 2). Altogether, these results suggest that only BisBAL NPs have a selective antitumor activity on CAL-27 cell line and compete in efficacy with commercial antitumor drugs. 


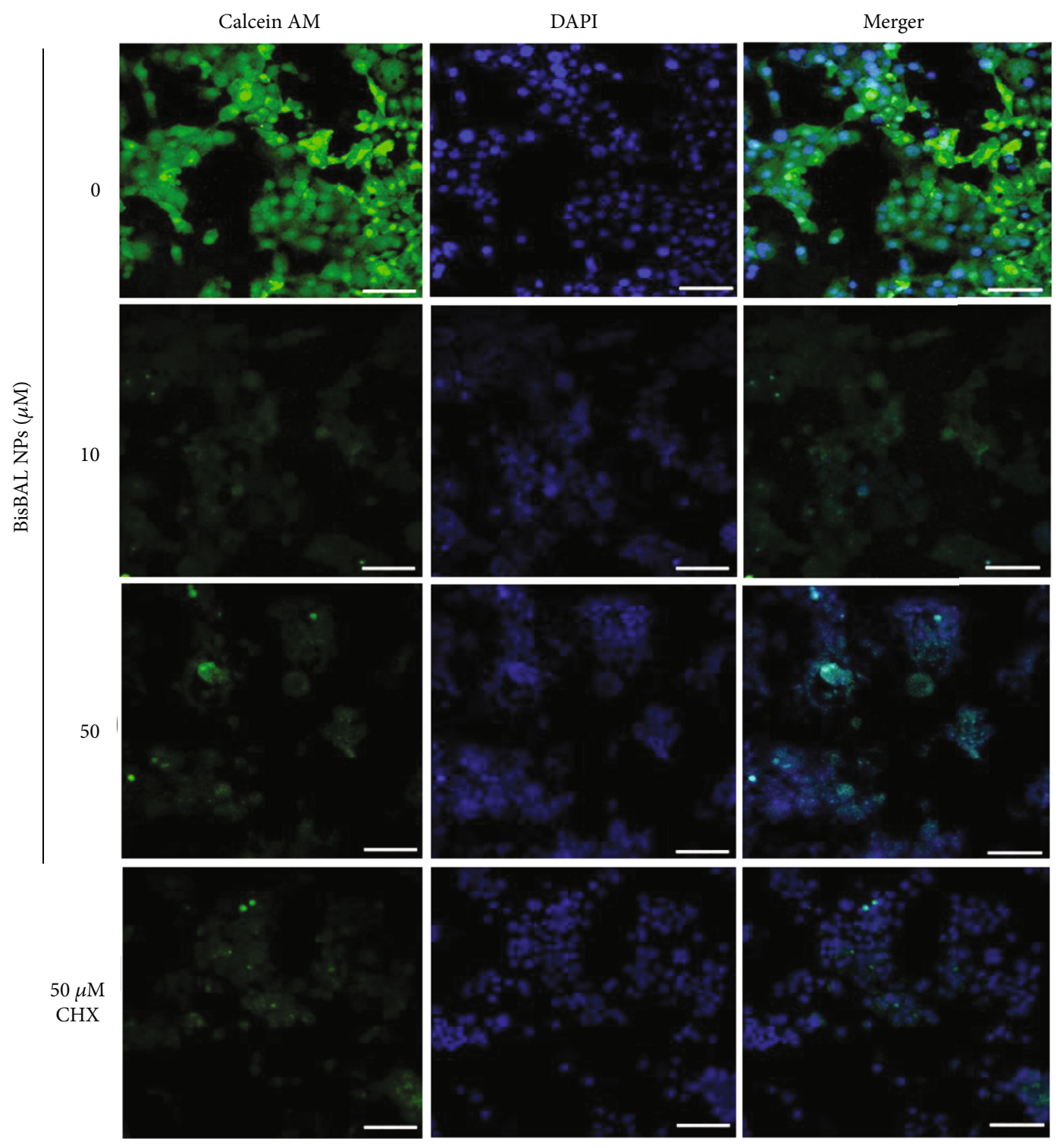

FIGURE 3: Cell membrane permeability of tumoral cells was determined after exposition to BisBAL NP or chlorhexidine by calcein-AM assay and fluorescence microscopy. CAL-27 cells were treated with 0,10 , or $50 \mu \mathrm{M}$ of BisBAL NP or $50 \mu \mathrm{M}$ of CHX for $24 \mathrm{~h}$. After exposition, cells were stained with calcein-AM and DAPI. Bar indicates $5 \mu \mathrm{m}$.

3.3. Cell Membrane Permeability Assays. To get an insight about the action mechanism of BisBAL NP and CHX on tumoral cells, cell membrane permeability assays were carried out to explore possible damage at the cell membrane of CAL-27 and HGF cells. After $24 \mathrm{~h}$ exposition to 0,10 , and $50 \mu \mathrm{M}$ of BisBAL NP and $50 \mu \mathrm{M}$ of CHX, cell morphology was observed by fluorescence microscopy. Tumoral cells showed clear damage after being exposed to $10 \mu \mathrm{M}$ of BisBAL NPs, and it was more evident when $50 \mu \mathrm{M}$ of BisBAL NPs were used (Figure 3). Similar results were obtained when tumoral cells were treated with $50 \mu \mathrm{M}$ of CHX (Figure 3). On the other side, HGFs treated with $0-50 \mu \mathrm{M}$ of BisBAL NP did not show evidence of cytotoxicity as can be seen in Figure 4. Fibroblasts look their typical long shape and also the nucleus lack signals of toxicity after exposition to 10$50 \mu \mathrm{M}$ of BisBAL NP. In contrast, $50 \mu \mathrm{M}$ of CHX showed a strong cytotoxic effect on HGFs (Figure 5), promoting calcein-AM release and amorphous nucleus. These data support our previous results about a selective antitumor activity of BisBAL NPs on human squamous cell carcinoma unlike $\mathrm{CHX}$.

3.4. DNA Ladder Assays. When the genotoxic effect of BisBAL NP and CHX was studied on tumoral cells, DNA ladder assays were developed. Our findings showed a typical ladder shape in the sample treated with $50 \mu \mathrm{M}$ of $\mathrm{CHX}$ (Figure 4, line 4). In contrast, DNA genomic exposed to $50 \mu \mathrm{M}$ of BisBAL NP did not show signals of degradation (Figure 4, line 3), presenting a band similar to DNA genomic control (Figure 4, line 2). These results suggest that only CHX and not BisBAL NP present a genotoxic effect on tumoral cells. 


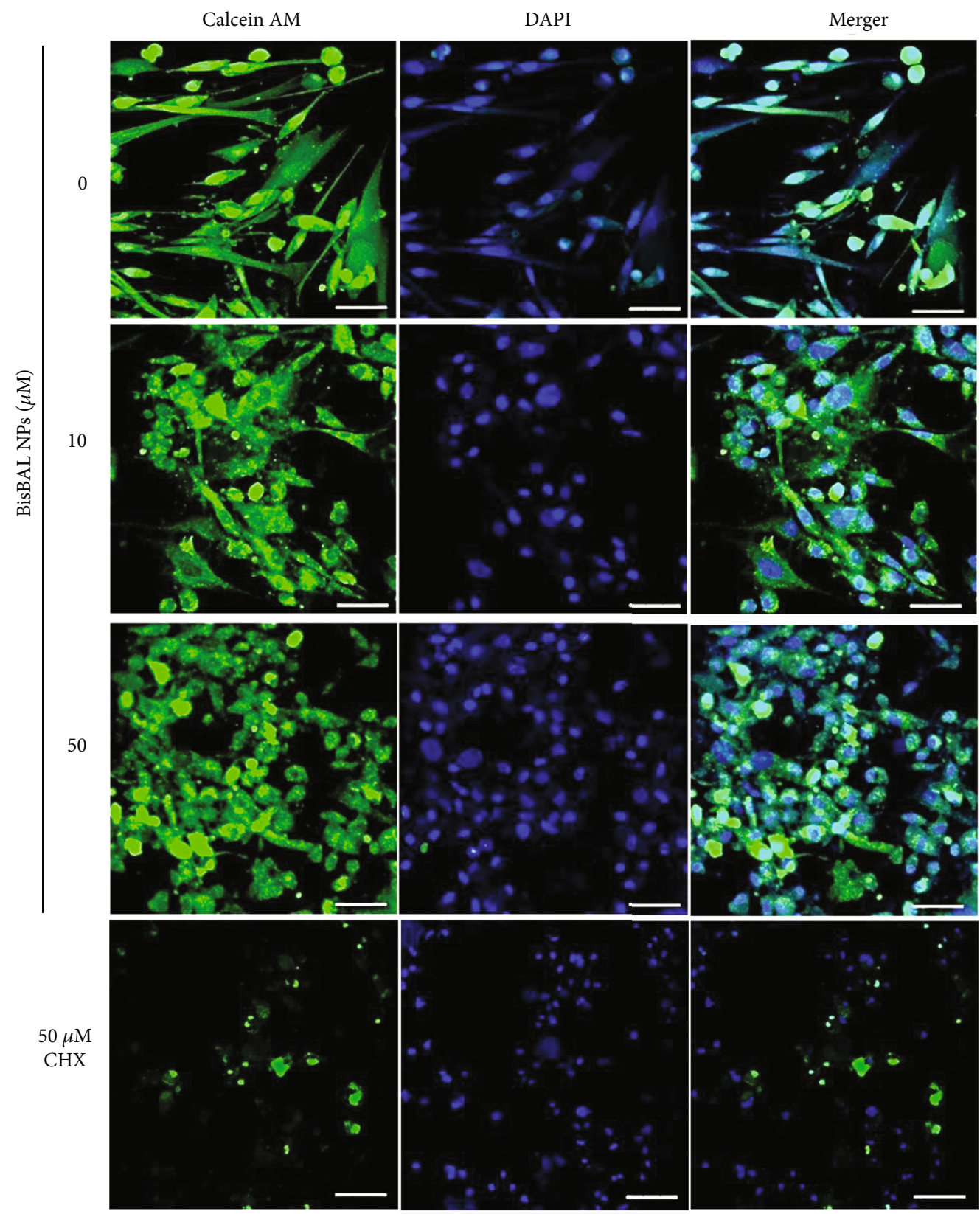

FIGURE 4: Cell membrane permeability of nontumoral control cells (HGFs) was determined after exposition to BisBAL NP or chlorhexidine by calcein-AM assay and fluorescence microscopy. HGF cells were treated with 0,10 , or $50 \mu \mathrm{M}$ of BisBAL NP or $50 \mu \mathrm{M}$ of CHX for $24 \mathrm{~h}$. After exposition, cells were stained with calcein-AM and DAPI. Bar indicates $5 \mu \mathrm{m}$.

\section{Discussion}

The first option of treatment for patients with oral cancer is surgery if it is possible [26]. However, the employ of chemotherapy and immunotherapy in oral cancer has increased recently. With the objective of reducing the incidence of metastatic recurrence, chemotherapy is advisable after surgery in most types of cancer [26]. In spite of good intentions, alone or in combination with radiation, chemotherapy can cause strong cytotoxic effects on nontumor cells of healthy tissue leading to cancer patients leaving treatment. Antitumor drugs like doxorubicin, docetaxel, and cisplatin have severe adverse side effects such as nephrotoxicity, bone marrow suppression, and cardiotoxicity. Early updates of metaanalysis evaluating the effectiveness of chemotherapy in the treatment of patients with oral cancer did not show benefit [27]. Therefore, it is urgent to develop selective and biocompatible anticancer drugs. Nanotechnology is a new area that explores biomolecular structures, developing "smart drugs" with a lot of applications in material science and medicine. Nanostructures are more reactive than common elements or molecules with a higher ratio of volume/area on their surface. Metal nanoparticles with different biological properties have been described like antibacterial, antimycotic, 


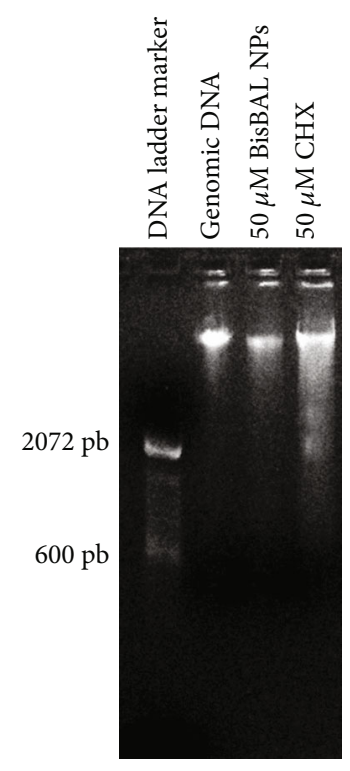

Figure 5: Effect of BisBAL NP and CHX on genomic DNA of CAL-27 cell line. Genomic DNA was extracted from tumoral cells exposed to $50 \mu \mathrm{M}$ of BisBAL NP or $50 \mu \mathrm{M}$ CHX for $24 \mathrm{~h}$. DNA was analyzed by electrophoresis in agarose gel and ethidium bromide staining.

antibiofilm, antitubercular, and antiviral activities [28, 29]. With antitumor activity, several metal nanoparticles have been reported including silver, iron, zinc, selenium, copper, iron, and vanadium $[18,19,30]$. In this study, we synthesized BisBAL NP by colloidal method and they were characterized by SEM like several early reports of our group [21, 22, 31, 32]. BisBAL NP showed the same circular shape, with an average size of $24 \mathrm{~nm}$, and agglomerates were evident in SEM images.

In this work, we tackle the lack of selectivity among antitumoral drugs and describe the antitumor activity of BisBAL NPs and CHX on human squamous cell carcinoma. Early reports have described the antitumor properties of $\mathrm{CHX}$ $[11,12]$; however, its antitumor activity was not compared with control nontumor cells at the same time to evaluate its selectivity against tumor cells. Our results show that CHX lacks selectivity inhibiting the growth of both tumor and control nontumor cells after $24 \mathrm{~h}$ of exposition at a final concentration of $25 \mu \mathrm{M}$. Previously, it was reported that $237.7 \mu \mathrm{M}$ of CHX inhibited $80 \%$ of AW 13516 cell (poorly to moderately differentiated squamous cell carcinoma of the tongue) growth [12]. In comparison with our results, we obtain higher inhibition of tumor cell growth with 10 times less concentration of CHX. Several previous studies have described the cytotoxic effect of CHX on different kinds of oral cells including fibroblasts and odontoblasts [13, 33-35]. Our results support these reports because $25 \mu \mathrm{M}$ of $\mathrm{CHX}$ interfered with $80 \%$ of human gingival fibroblast employed as noncancer cells. BisBAL NPs inhibit $90 \%$ of the tumor cell growth when $25 \mu \mathrm{M}$ was added to the cultured cells. This datum agreed with our early report employing the same nanoclusters on breast cancer cells [21]. Early reports have been described the antitumor activity of silver nanoparticles $[36,37]$; however, they cannot evaluate their effect on control nontumor cells at the same time. Unlike CHX, BisBAL NPs showed a selective antitumor activity with more than $80 \%$ of cell viability of noncancer cells at $25 \mu \mathrm{M}$, suggesting that BisBAL NPs are an innovative alternative to tackle the lack of selectivity among antitumor drugs.

The action mechanism of BisBAL NPs to inhibit the tumoral cell growth seems to be based on their lipophilic properties. Our experiments with calcein-AM showed clear damage in the cell plasmatic membrane of tumoral cells post-treatment with BisBAL NPs leading to cell lysis. Tumoral cells exposed to CHX presented similar damage in their membrane, affecting their permeability. We hypothesize that the lipophilic character of BisBAL NP is associated with their selective effect on tumoral cells. Early reports or our group described an identical phenomenon with breast cancer cells [21]. Employing higher concentrations of BisBAL NPs could increase the damage of the cell membrane, thus promoting faster cell lysis. Interestingly, the genomic DNA of tumoral cells was not damaged after $24 \mathrm{~h}$ exposition with $50 \mu \mathrm{M}$ of BisBAL NPs. In contrast, DNA of tumoral cells treated with $50 \mu \mathrm{M}$ CHX showed a clear ladder shape, indicating a typical genotoxic effect. Altogether, these results suggest that CHX kill both tumoral and nontumoral control cells in the same way; altering their cell membrane permeability and damaging their genomic DNA lacking selectivity. Several early studies have been described as the cytotoxicity of CHX on different kinds of cells [13, 33, 38]. Previously, it was reported that $1 \%$ of $\mathrm{CHX}$ shows a cytotoxic effect on human gingival fibroblasts (same control cells used in our study) employing also MTT cell viability assays [39]. Also, it has been described that cytotoxicity and genotoxicity of $\mathrm{CHX}$ in a dose-dependent phenomenon on macrophages may be via ROS generation [40].

It is important to underline the low cost of synthesis of BisBAL NPs in comparison with the high cost of common antitumor drugs [41, 42], because, in developing countries, the cost of chemotherapy treatment is an important cause of leaving the treatment [43]. We estimate that bismuth nanoparticle synthesis is 140 times less expensive in comparison with doxorubicin or docetaxel, two of the most common antitumor drugs.

In conclusion, we present evidence of the effectiveness of a BisBAL NP hydrogel as a selective growth inhibitor of human cervix uterine, prostate, and colorectal cancer cell lines. BisBAL NP-loaded hydrogels may be a low-cost, innovative alternative for the topical treatment of cervical, prostate, and colon cancer without adverse effects on nontumor cells.

\section{Data Availability}

The data that support the findings of this study are available from the corresponding author, Claudio Cabral-Romero, upon reasonable request.

\section{Conflicts of Interest}

The authors declare that there are no conflicts of interest. 


\section{Acknowledgments}

This study was an approved project by the Sectorial Fund for Education Research, CONACyT (CB2017-2018 and A1-S-20148). The authors want to thank to Nayely Pineda-Aguilar from CIMAV-Unidad Monterrey for their technical assistance in SEM analysis of BisBAL NPs.

\section{References}

[1] S. Warnakulasuriya, "Global epidemiology of oral and oropharyngeal cancer," Oral Oncology, vol. 45, no. 4-5, pp. 309-316, 2009.

[2] F. Bray, J. Ferlay, I. Soerjomataram, R. L. Siegel, L. A. Torre, and A. Jemal, "Global cancer statistics 2018: GLOBOCAN estimates of incidence and mortality worldwide for 36 cancers in 185 countries," CA: a Cancer Journal for Clinicians, vol. 68, no. 6, pp. 394-424, 2018.

[3] K. Pollaers, O. Kujan, N. W. Johnson, and C. S. Farah, "Oral and oropharyngeal cancer in Oceania," Translational Research in Oral Oncology, vol. 2, p. 2057178X1772645, 2017.

[4] N. W. Johnson, S. Warnakulasuriya, P. C. Gupta et al., "Global oral health inequalities in incidence and outcomes for oral cancer: causes and solutions," Advances in Dental Research, vol. 23, no. 2, pp. 237-246, 2011.

[5] A. V. Nascimento, A. Singh, H. Bousbaa, D. Ferreira, B. Sarmento, and M. M. Amiji, "Overcoming cisplatin resistance in non-small cell lung cancer with _Mad2_ silencing siRNA delivered systemically using EGFR-targeted chitosan nanoparticles," Acta Biomaterialia, vol. 47, pp. 71-80, 2017.

[6] U. Kilic, K. Sahin, M. Tuzcu et al., "Enhancement of cisplatin sensitivity in human cervical cancer: epigallocatechin-3-gallate," Frontiers in Nutrition, vol. 1, 2015.

[7] R. Kitagawa, N. Katsumata, T. Shibata et al., "Paclitaxel plus carboplatin versus paclitaxel plus cisplatin in metastatic or recurrent cervical cancer: the open-label randomized phase III trial JCOG0505," Journal of Clinical Oncology, vol. 33, no. 19, pp. 2129-2135, 2015.

[8] J. A. Yared and K. H. Tkaczuk, "Update on taxane development: new analogs and new formulations," Drug Design, Development and Therapy, vol. 6, pp. 371-384, 2012.

[9] Z. Wang, X. Liang, J. Yu et al., "Non-genetic risk factors and predicting efficacy for docetaxel-drug-induced liver injury among metastatic breast cancer patients," Journal of Gastroenterology and Hepatology, vol. 27, no. 8, pp. 1348-1352, 2012.

[10] H. K. Park, J. E. Lee, J. Lim et al., "Combination treatment with doxorubicin and gamitrinib synergistically augments anticancer activity through enhanced activation of Bim," BMC Cancer, vol. 14, no. 1, 2014.

[11] K. G. Park, U. Chetty, W. Scott, and W. Miller, "The activity of locally applied cytotoxics to breast cancer cells in vitro," Annals of the Royal College of Surgeons of England, vol. 73, no. 2, pp. 96-99, 1991.

[12] M. R. Khairnar, U. Wadgave, H. Jadhav, and R. Naik, "Anticancer activity of chlorhexidine and cranberry extract: an invitro study," Journal of Experimental Therapeutics \& Oncology, vol. 12, no. 3, pp. 201-205, 2018.

[13] L. B. de Souza, S. G. de Aquino, P. P. de Souza, J. Hebling, and C. A. Costa, "Cytotoxic effects of different concentrations of chlorhexidine," American Journal of Dentistry, vol. 20, no. 6, pp. 400-404, 2007.
[14] M. Graber, M. Hell, C. Grost et al., "Oral Disinfectants Inhibit Protein-Protein Interactions Mediated by the Anti-Apoptotic Protein Bcl-xL and Induce Apoptosis in Human Oral Tumor Cells," Angewandte Chemie (International Ed. in English), vol. 52, no. 16, pp. 4487-4491, 2013.

[15] J. Shi, P. W. Kantoff, R. Wooster, and O. C. Farokhzad, "Cancer nanomedicine: progress, challenges and opportunities," Nature Reviews Cancer, vol. 17, no. 1, pp. 20-37, 2017.

[16] M. Cordani and A. Somoza, "Targeting autophagy using metallic nanoparticles: a promising strategy for cancer treatment," Cellular and Molecular Life Sciences, vol. 76, no. 7, pp. 1215-1242, 2019.

[17] B. Garcia-Pinel, C. Porras-Alcala, A. Ortega-Rodriguez et al., "Lipid-based nanoparticles: application and recent advances in cancer treatment," Nanomaterials, vol. 9, no. 4, p. 638, 2019.

[18] S. Menon, S. D. KS, S. R, R. S, and V. K. S, "Selenium nanoparticles: a potent chemotherapeutic agent and an elucidation of its mechanism," Colloids and Surfaces B: Biointerfaces, vol. 170, pp. 280-292, 2018.

[19] A. Krol, P. Pomastowski, K. Rafinska, V. Railean-Plugaru, and B. Buszewski, "Zinc oxide nanoparticles: synthesis, antiseptic activity and toxicity mechanism," Advances in Colloid and Interface Science, vol. 249, pp. 37-52, 2017.

[20] G. Shim, M. G. Kim, D. Kim, J. Y. Park, and Y. K. Oh, "Nanoformulation-based sequential combination cancer therapy," Advanced Drug Delivery Reviews, vol. 115, pp. 57-81, 2017.

[21] R. Hernandez-Delgadillo, C. M. Garcia-Cuellar, Y. SanchezPerez et al., "In vitro evaluation of the antitumor effect of bismuth lipophilic nanoparticles (BisBAL NPs) on breast cancer cells," International Journal of Nanomedicine, vol. 13, pp. 6089-6097, 2018.

[22] A. R. Badireddy, R. Hernandez-Delgadillo, R. I. SánchezNájera, S. Chellam, and C. Cabral-Romero, "Synthesis and characterization of lipophilic bismuth dimercaptopropanol nanoparticles and their effects on oral microorganisms growth and biofilm formation," Journal of Nanoparticle Research, vol. 16, no. 6, 2014.

[23] Y. Liu, D. A. Peterson, H. Kimura, and D. Schubert, "Mechanism of cellular 3-(4,5-dimethylthiazol-2-yl)-2,5-diphenyltetrazolium bromide (MTT) reduction," Journal of Neurochemistry, vol. 69, no. 2, pp. 581-593, 1997.

[24] H.-D. Rene, A. R. Badireddy, M. S. Juan José et al., "Cytotoxic effect of lipophilic bismuth dimercaptopropanol nanoparticles on epithelial cells," Journal of Nanoscience and Nanotechnology, vol. 16, no. 1, pp. 203-209, 2016.

[25] A. Ogata, S. Mitsui, H. Yanagie et al., "A novel anti-tumor agent, polyoxomolybdate induces apoptotic cell death in AsPC-1 human pancreatic cancer cells," Biomedicine \& Pharmacotherapy, vol. 59, no. 5, pp. 240-244, 2005.

[26] L. Hartner, "Chemotherapy for oral cancer," Dental Clinics of North America, vol. 62, no. 1, pp. 87-97, 2018.

[27] J. P. Pignon, A. . Maître, E. Maillard, and J. Bourhis, "Metaanalysis of chemotherapy in head and neck cancer (MACHNC): an update on 93 randomised trials and 17,346 patients," Radiotherapy and Oncology, vol. 92, no. 1, pp. 4-14, 2009.

[28] A. Grumezescu and C. Chifiriuc, "Editorial (Thematic Issue: Prevention of microbial biofilms - the contribution of micro and nanostructured Materials)," Current Medicinal Chemistry, vol. 21, no. 29, p. 3311, 2014.

[29] F. N. Oktar, M. Yetmez, D. Ficai, A. Ficai, F. Dumitru, and A. Pica, "Molecular mechanism and targets of the antimicrobial 
activity of metal nanoparticles," Current Topics in Medicinal Chemistry, vol. 15, no. 16, pp. 1583-1588, 2015.

[30] I. E. Leon, J. F. Cadavid-Vargas, A. L. Di Virgilio, and S. B. Etcheverry, "Vanadium, ruthenium and copper compounds: a new class of nonplatinum metallodrugs with anticancer activity," Current Medicinal Chemistry, vol. 24, no. 2, pp. 112-148, 2017.

[31] R. Hernandez-Delgadillo, A. R. Badireddy, V. ZaragozaMagaña, R. I. Sánchez-Nájera, S. Chellam, and C. CabralRomero, "Effect of lipophilic bismuth nanoparticles on erythrocytes," Journal of Nanomaterials, vol. 2015, Article ID 264024, 9 pages, 2015.

[32] R. Hernandez-Delgadillo, C. Del Angel-Mosqueda, J. M. Solis-Soto et al., "Antimicrobial and antibiofilm activities of MTA supplemented with bismuth lipophilic nanoparticles," Dental Materials Journal, vol. 36, no. 4, pp. 503-510, 2017.

[33] S. Balloni, P. Locci, A. Lumare, and L. Marinucci, "Cytotoxicity of three commercial mouthrinses on extracellular matrix metabolism and human gingival cell behaviour," Toxicology In Vitro, vol. 34, pp. 88-96, 2016.

[34] A. J. Mariotti and D. A. Rumpf, "Chlorhexidine-induced changes to human gingival fibroblast collagen and non-collagen protein production," Journal of Periodontology, vol. 70, no. 12, pp. 1443-1448, 1999.

[35] F. C. Lessa, A. M. Aranha, I. Nogueira, E. M. Giro, J. Hebling, and C. A. Costa, "Toxicity of chlorhexidine on odontoblastlike cells," Journal of Applied Oral Science, vol. 18, no. 1, pp. 50-58, 2010.

[36] W. Chengzheng, W. Jiazhi, C. Shuangiiang et al., "Biogenic synthesis, characterization and evaluation of silver nanoparticles from Aspergillus niger JX556221 against human colon cancer cell line HT-29," Journal of Nanoscience and Nanotechnology, vol. 18, no. 5, pp. 3673-3681, 2018.

[37] E. S. Al-Sheddi, N. N. Farshori, M. M. Al-Oqail et al., “Anticancer potential of green synthesized silver nanoparticles using extract of Nepeta deflersiana against human cervical cancer cells (HeLA)," Bioinorganic Chemistry and Applications, vol. 2018, Article ID 9390784, 12 pages, 2018.

[38] J. X. Liu, J. Werner, T. Kirsch, J. D. Zuckerman, and M. S. Virk, "Cytotoxicity evaluation of chlorhexidine gluconate on human fibroblasts, myoblasts, and osteoblasts," Journal of Bone and Joint Infection, vol. 3, no. 4, pp. 165-172, 2018.

[39] U. P. Verma, A. Gupta, R. K. Yadav, R. Tiwari, R. Sharma, and A. K. Balapure, "Cytotoxicity of chlorhexidine and neem extract on cultured human gingival fibroblasts through fluorescence-activated cell sorting analysis : an in-vitro study," European Journal of Dentistry, vol. 12, no. 3, pp. 344-349, 2018.

[40] Y. C. Li, Y. H. Kuan, S. S. Lee, F. M. Huang, and Y. C. Chang, "Cytotoxicity and genotoxicity of chlorhexidine on macrophages in vitro," Environmental Toxicology, vol. 29, no. 4, pp. 452-458, 2014.

[41] V. Prasad, K. De Jesus, and S. Mailankody, "The high price of anticancer drugs: origins, implications, barriers, solutions," Nature Reviews. Clinical Oncology, vol. 14, no. 6, pp. 381390, 2017.
[42] G. Dranitsaris, X. Zhu, G. Adunlin, and M. D. Vincent, "Cost effectiveness vs. affordability in the age of immuno-oncology cancer drugs," Expert Review of Pharmacoeconomics \& Outcomes Research, vol. 18, no. 4, pp. 351-357, 2018.

[43] A. Pichon-Riviere, O. U. Garay, F. Augustovski et al., "Implications of global pricing policies on access to innovative drugs: the case of trastuzumab in seven Latin American countries," International Journal of Technology Assessment in Health Care, vol. 31, no. 1-2, pp. 2-11, 2015. 


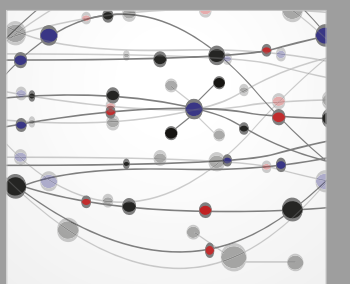

The Scientific World Journal
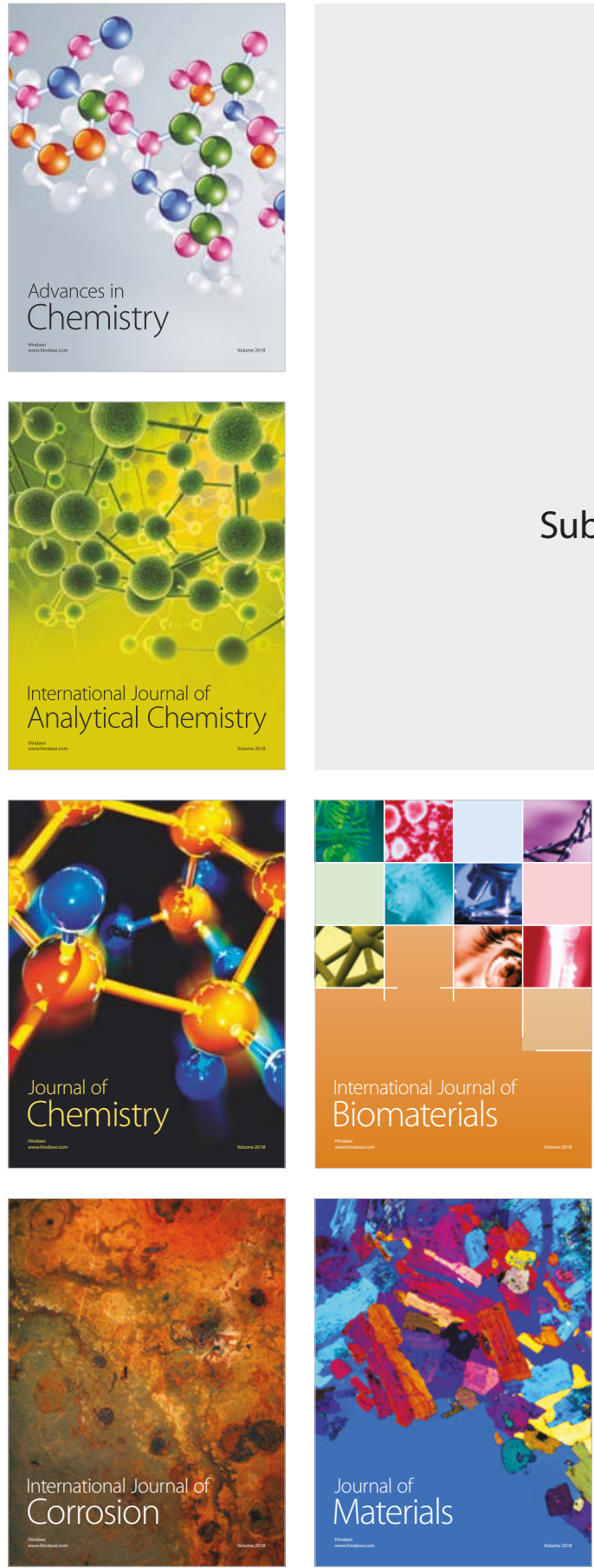

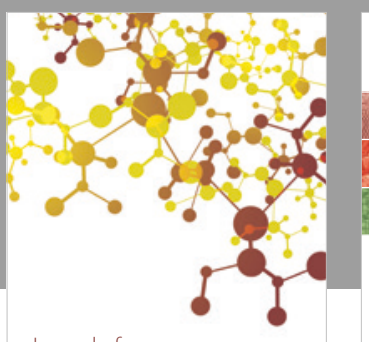

Journal of

Applied Chemistry
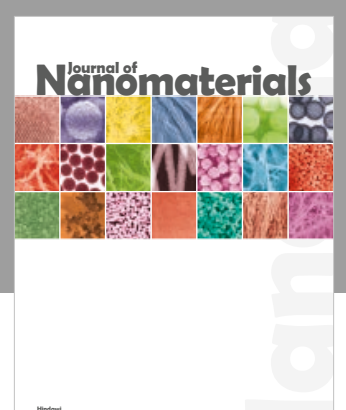

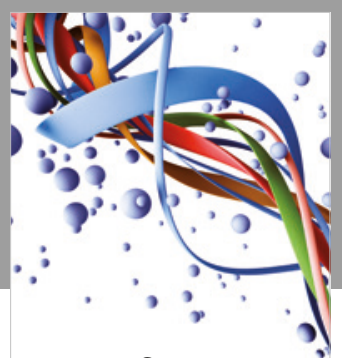

Scientifica

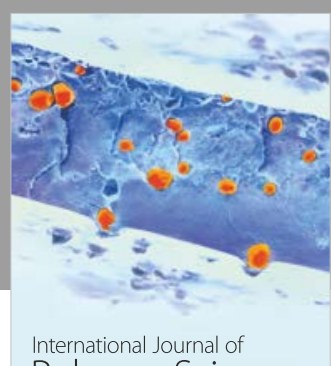

Polymer Science

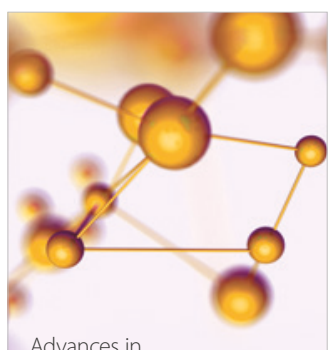

Physical Chemistry
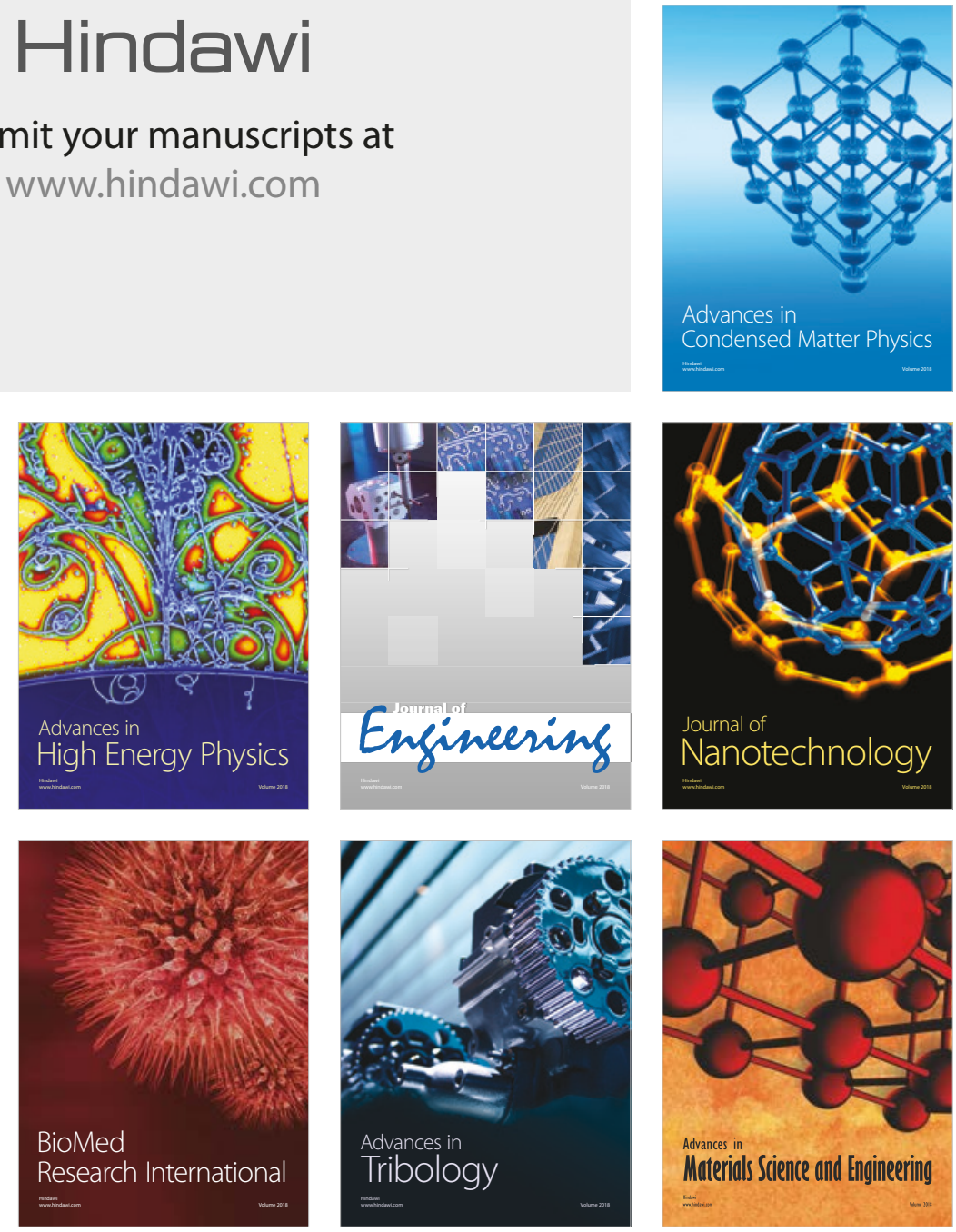